\title{
Lorsqu'il s'agit de se taire. Réflexions sur l'écriture en compagnie de Maurice Blanchot
}

\author{
Florence Vandendorpe \\ Université catholique de Louvain
}

Pour Blanchot, l'écriture est un mouvement par lequel l'écrivain, s'exilant de lui-même, se rend disponible à une parole qui n'est pas de lui ${ }^{1}$. Par-delà le visible, à l'abri du monde où les choses sont réduites à ce que l'on peut en faire, cela parle. Et grâce à l'écriture, qui lui permet d'entrer en relation avec ce

\footnotetext{
1 Une version préliminaire de cet article a fait l'objet d'une communication intitulée "Écrire. Entre présence et absence, un espace s'ouvre », lors du colloque Image / Imaginaire dans l'œuvre de Maurice Blanchot organisé par l'Université Paris 10 à l'occasion du Centenaire de Maurice Blanchot (Nanterre, 27 et 28 avril 2007).
} 
quelque chose qui parle, l'écrivain peut l'entendre. L'écriture, en quelque sorte, est un pont vers une autre dimension de l'être. À travers elle, l'écrivain se place en position de médiateur entre deux univers : le monde, d'une part, où règnent l'utopie et l'illusion; et le réel, d'autre part, insaisissable, où se trouve la vraie profondeur des choses. Lorsqu'on parcourt son œuvre, on ne peut manquer d'être frappé par la place importante qu'y occupe cet autre espace - pour autant que l'on puisse ainsi nommer ce qui, nous le verrons, relève pour lui d'une expérience avant tout - et par la diversité des regards qu'il a posés sur lui. Il écrit, dans L'espace littéraire, que l'œuvre littéraire, parce qu'elle nous met en relation avec cet au-delà du monde que la connaissance ignore, entretient un rapport privilégié avec le vrai :

Dans l'œuvre d'art, l'être se risque, car tandis que dans le monde où les êtres le repoussent pour être il est toujours dissimulé, nié et renié (en ce sens, aussi, protégé), là, en revanche, où règne la dissimulation, ce qui se dissimule tend à émerger dans le fond de l'apparence, ce qui est nié devient le trop-plein de l'affirmation, - mais apparence qui, cependant, ne révèle rien, affirmation où rien ne s'affirme, qui est seulement la position instable à partir de quoi, si l'œuvre réussit à la contenir, le vrai pourra avoir lieu. (1955, p. 324)

Il dit également, dans Le pas au-delà, qu'« écrire [...], c'est toujours d'abord récrire, et récrire ne renvoie à aucune écriture préalable, pas plus qu'à une antériorité de parole ou de présence ou de signification " (1973, p. 48). Surprenant discours, où des énoncés par ailleurs contradictoires sont mis côte à côte comme s'il importait, pour rendre compte de ce qui est en jeu, de dépasser les oppositions; comme si ce dont il est question relevait d'un ordre des choses où les contradictions n'ont plus cours. Je propose de développer différents propos 
sur l'écriture que l'on trouve dans l'œuvre de Blanchot en me penchant, précisément, sur cette tension caractéristique de sa pensée. Celle-ci, nous le verrons, s'articule autour d'une tension entre présence et absence, parole et silence. Je m'attarderai plus particulièrement sur le premier pôle : celui de la présence, c'est-à-dire de l'ouverture du créateur à quelque chose qui lui vient d'ailleurs. Dans les études consacrées à l'œuvre de Blanchot, celui-ci, en effet, me semble étonnamment peu développé (voir Collin, 1971, et Antonioli, 1999).

Cette réflexion sur le processus d'écriture nous conduira, dans un second temps, à nous pencher sur ce qui distingue création et expression. Alors que les ateliers d'expression artistique à des fins thérapeutiques se multiplient comme des petits pains et investissent des terrains de plus en plus diversifiés, il est sans doute pertinent de s'interroger sur ce dont l'œuvre d'art est l'écho. La réflexion de Blanchot, qui rejoint la manière dont des artistes et des psychanalystes abordent la création aujourd'hui, nous servira de guide sur ce terrain.

Les ouvrages sur lesquels s'appuie ma réflexion sont L'espace littéraire (1955), Le livre à venir (1959), Le pas au-delà (1973), L'Écriture du désastre (1980) et Une voix venue d'ailleurs (2002 [1992]), soit des textes critiques. À la suite de Dominique Rabaté (2005), je considère que, dans ces ouvrages, le propos de Blanchot consiste à rendre compte d'une expérience bien plus qu'à défendre une position théorique. Je les aborderai donc comme autant de témoignages de la manière dont il appréhende l'écriture. 


\section{Lorsqu'il s'agit de se taire}

Pour Blanchot, le silence et l'écriture sont intimement liés. C'est dans le silence que l'écriture peut naître, mais c'est aussi sous la forme d'un certain silence, nous le verrons, qu'elle se donne à lire. Un silence qui se décline à plusieurs niveaux.

Il y a, tout d'abord, le silence de l'écrivain. L'œuvre, écrit Blanchot, « exige de l'écrivain qu'il perde toute "nature", tout caractère, et que, cessant de se rapporter aux autres et à luimême par la décision qui le fait moi, il devienne le lieu vide où s'annonce l'affirmation impersonnelle » (1955, p. 58). L'écriture, pour lui, prend place en dehors du champ de la subjectivité. Nous verrons plus loin en quoi consiste l'affirmation impersonnelle qu'il évoque ici. Relevons simplement que celle-ci, pour Blanchot, ne peut être entendue qu'à condition que l'écrivain mette en suspens, pour un temps, la conscience qu'il a de lui-même. Il s'agit qu'il se retire, qu'il (s')oublie. Comme l'a exprimé Jean-Luc Lannoy, l'oubli, pour Blanchot, " est contemporain d'une dimension d'accueil et d'adhésion, entrée dans l'étendue essentielle, libre communication entre l'intimité des choses et le soi percevant. » (2005, p. 184).

Cette exigence de retrait, d'exil de l'écrivain à l'égard de lui-même, nombreux sont les créateurs qui en ont témoigné. Jean Florence, se référant au travail du peintre, l'exprime en ces mots :

Le peintre fait silence; il fait taire le bruissement continu de ce que Kant appelle la « rhapsodie des sensations », il crée un lieu de concentration, de condensation, où le silence est imposé aux significations bavardes de l'expérience. Une pause. (1985, p. 139) 
Ce mouvement par lequel l'écrivain se dérobe à lui-même et aux sollicitations du monde n'est pas dépourvu de risques. En témoignent les nombreuses associations entre l'écriture et la mort que l'on trouve dans les écrits de Blanchot, comme celle-ci : «L'œuvre est elle-même une expérience de la mort dont il semble qu'il faille disposer préalablement pour parvenir à l'œuvre et, par l'œuvre, à la mort. » (1955, p. 111) Ce mouvement de retrait, nous dit-il, livre en effet l'écrivain sans défense à « la toute-puissance solitaire de la fascination » (1955, p. 53). Dans cette quête de l'insaisissable qu'est l'écriture, il se retrouve seul, exilé de lui-même et du monde, absorbé par le mouvement qui le projette en avant de lui-même dans une tentative jamais aboutie de se fondre dans la présence pleine de l'instant.

De nombreux créateurs ont témoigné de cette expérience forte, totale, qu'est la création. Pour le peintre Jean Bazaine, celle-ci relève de la passion :

Être au monde, pour un peintre, c'est se laisser consumer par lui.

Son difficile effort d'être, ce sera de fixer sans relâche une réalité qui l'aveugle, vide de toute pensée, ivre seulement d'une chasse à l'impossible, dans la passion d'une attente sans fin, dans la seule conscience claire et acceptée de l'insoluble. (1973, p. 96)

Alain Didier-Weill a souligné la dimension jouissive de cet élan de l'artiste vers quelque chose qui l'appelle. Ce moment, écrit-il, marque le début du processus créateur et témoigne des dangers auxquels, comme toute forme de jouissance, il est subjectivement associé : « Le danger tient à la possibilité qu'a le sujet chantant, guidé par l'espoir de trouver la source de son âme au ciel, d'y rencontrer effectivement cette source : 
l'intensité de l'extase risque alors d'être telle qu'il peut décider de vouloir rester dans ce "haut" lieu de jouissance et de ne pas redescendre ici-bas. » (2003, p. 33) C'est du chant qu'Alain Didier-Weill parle ici, mais on se doute que ses mots valent également pour les autres activités créatrices. On sait en effet, depuis les travaux d'Anzieu (1981), combien ce sentiment de dépersonnalisation, de traversée d'une expérience qui comporte un risque mortel, est caractéristique de la première phase de tout travail de création. Phase qu'il nomme d'ailleurs, non sans raison, celle du « saisissement créateur » (1981, p. 96). Celle-ci, nous dit-il, est de nature psychotique, même si elle n'est pas pathologique pour autant. Il s'agit d'une expérience angoissante, vécue comme vitale, durant laquelle le créateur a le sentiment, justifié sur le plan psychique, que l'unité de sa personne est menacée.

L'écriture, donc, pour Blanchot, est un mouvement par lequel l'écrivain se rend disponible à une parole qu'il ressent comme ne venant pas de lui, à laquelle il prête sa voix. C'est là ce que nous pourrions appeler son contexte d'émergence. Nous sommes très loin, ici, de l'image de l'écrivain qui, par l'écriture, affirme ce qu'il est à la face du monde. Nous y reviendrons.

\section{Une parole pour horizon}

Il y aurait ainsi, au départ de l'œuvre littéraire, et plus généralement au départ de toute œuvre artistique, l'irruption de quelque chose dans l'horizon du créateur. Jean Florence, dans un texte consacré à la peinture, décrit ce moment comme « une trouée dans le monde visible» :

Il y aurait ainsi, au commencement, lequel commencement ne se donnant jamais qu'après coup, une trouée dans le monde 
visible; l'éclat d'un regard traversant l'espace de la vision. Par cette fenêtre ouverte, une autre réalité vient s'offrir qui contraint à peindre, qui appelle le tableau à naître pour répondre à ce regard, pour répondre de ce regard. Le travail du peintre ne serait pas commandé par la réalité donnée dans la vision qu'il s'agirait de reproduire par l'artifice de la couleur, de la ligne, de la luminosité mais serait régi par l'exigence de donner réponse à ce moment de vide, à ce point d'origine audelà du plan de la vision. (1985, p. 138)

Pour Henry Bauchau, cela prend la forme de sensations : " Cela commence par un son, un rythme, une image [...]. Je ne sais d'où surviennent ces sensations inattendues. " (2000, p. 29) Blanchot, nous l'avons vu, parle quant à lui d'une parole. Tous trois s'accordent pour aborder l'inspiration comme un moment durant lequel quelque chose se manifeste à l'auteur : cela parle.

Cela parle... Et cependant personne, affirme Blanchot, ne parle : cette parole est impersonnelle. L'écriture, comme l'écrit Dominique Rabaté, est pour lui « le passage du Je au Il [...]. Cette transformation du sujet en un pronom neutre, c'est sans doute cela même l'épreuve particulière de l'écriture, celle que chaque écrivain doit rejouer pour son compte, en lui donnant provisoirement et à jamais son nom, en donnant à ce mouvement ce nom qu'il efface du même coup. » (2005, p. 1011) Écrire, en d'autres mots, est un geste par lequel l'écrivain se livre à un temps hors du temps où cela parle à travers lui.

Nous entrons, ici, dans ce qui pour Blanchot caractérise l'imaginaire. Ce mouvement à travers lequel quelque chose se dit prend, dans ses écrits, la forme d'une rencontre. Celui qui écrit, par l'acte même d'écrire, prête sa voix à une mémoire atemporelle, incessante, à laquelle il s'offre en réceptacle afin de pouvoir en témoigner. Cette parole qui se fait entendre, 
Blanchot la décrit comme une " écriture blanche, absente et neutre » (1959, p. 255), ou comme "l'affirmation ininterrompue, le murmure géant [...], profondeur parlante, indistincte plénitude qui est vide »(1955, p. 18). Relevons, dans ces extraits, la tension suscitée par la présence de termes qui évoquent tantôt le vide, tantôt le plein. Si Blanchot, par moments, insiste sur le caractère inaudible, creux, de cette parole autour de laquelle son écriture tourne et retourne comme une abeille sur une fleur, il ne cesse pour autant d'insister sur le caractère plein, sidérant, de ce silence qui se fait parole. La parole qui se laisse entendre dans l'imaginaire cumule, pour lui, deux qualités à première vue contradictoires : celle, d'une part, d'être creuse; celle, d'autre part, d'être parole. Deux dimensions intimement mêlées qui, dans ses écrits, sont constamment évoquées. Il insiste tantôt sur l'une, tantôt sur l'autre, sans jamais évacuer cette dualité qui, pour lui, est manifestement fondamentale. Si l'on veut rendre compte de sa pensée, on ne peut faire l'impasse sur celle-ci que nous allons, à présent, développer.

\section{Derrière les mots, le vide}

Commençons par ce que j'appellerai le côté du vide : l'idée, défendue par Blanchot dans ses écrits, que la parole qui vient à l'écrivain ne renvoie à rien. Elle ne renvoie à rien tout simplement parce qu'elle ne repose sur rien : elle est sans fondement. Et puisque c'est sur cette parole qu'elle repose, l'écriture, à son tour, " ne renvoie à aucune écriture préalable, pas plus qu'à une antériorité de parole ou de présence ou de signification ». (1973, p. 48) En d'autres mots, ce que l'écrivain écrit n'existe pas en dehors de l'écrit. L'œuvre ne dit rien. C'est 
en son mouvement d'être (en) œuvre qu'elle trouve et sa source et son centre. Sa réalité d'œuvre en train de se faire la définit, en un point jamais atteint puisque lorsqu'elle s'y confond elle cesse, par là-même, d'être œuvre. L'écriture, en d'autres mots, est un espace où rien ne peut se lire sinon le mouvement du récit qui se déploie. On trouve cette idée, notamment, dans Le livre à venir :

L'au-delà de l'œuvre n'est réel que dans l'œuvre, n'est que la réalité propre de l'œuvre. Le récit, par ses mouvements de caractère labyrinthique ou par la rupture de niveau qu'il produit dans sa substance, semble attiré hors de lui-même par une lumière dont nous croyons surprendre çà et là le reflet, mais cet attrait qui le déporte vers un point infiniment extérieur, est le mouvement qui le reporte vers le secret de luimême, vers son centre, vers l'intimité à partir de laquelle toujours il s'engendre et est sa propre et éternelle naissance. (1959, p. 112)

Cette approche de la création comme processus rappelle, bien sûr, la phénoménologie. Elle évoque, également, les travaux de Loreau : pour ce dernier, il n'est pas possible de distinguer arbitrairement un réel que le créateur chercherait à traduire et une forme à travers laquelle ce réel se laisserait lire dans une œuvre. Ce que l'œuvre donne à voir ne lui préexiste pas, car c'est elle-même qui le crée : « Le peintre trace; faisant avancer son pinceau, il ne trace rien d'autre que le tracement et ce tracement est un processus d'apparition, de pure apparition, d'apparition de soi : rien de plus encore. » (1998, p. 189)

Cette approche de l'écriture comme mouvement ne tendant vers rien d'autre que vers lui-même coexiste, dans l'œuvre de Blanchot, avec des affirmations qui, au contraire, conduisent à envisager l'œuvre d'art comme la trace de quelque chose qui existe par ailleurs et dont le créateur tente de se faire 
l'écho. Quelque chose qui, toutefois, toujours lui échappe. Pour Blanchot, lorsque le monde apparaît à celui qui écrit, il se dérobe en effet en même temps : " tout est parole, mais [...] la parole n'est plus elle-même que l'apparence de ce qui a disparu » (1955, p. 42), " l'image est l'absence de l'objet » (1955, p. 46). Se dégage ici l'idée d'un réel en retrait, auquel l'imaginaire ne livre qu'un accès périmé. S'enracinant dans le monde commun dont il s'efforce de faire taire les sollicitations constantes, l'écrivain s'approche, par l'écriture, de ce qui reste du monde lorsqu'on en a enlevé le côté contingent. Les images qui lui parviennent sont autant d'empreintes de quelque chose qui certes fut là, mais qui n'y est plus. Comme lorsque croyant tenir quelqu'un par la main, on réalise que l'on ne tient qu'un gant. Le réel, en quelque sorte, ne livre que son ombre : il déborde toujours des formes, des mots à travers lesquels il se donne à voir. Il est " ce qu'il y a de plus discontinu, l'intermittence de ces instants de lumière d'où lui vient la possibilité d'écrire » (1959, p. 30), et quand bien même il aurait le sentiment de l'avoir touché du doigt, l'écrivain, qui pour en témoigner ne peut s'appuyer que sur des structures de sens préalablement définies, est immanquablement condamné à l'échec. En effet, « qu'est-ce qui reste de la présence lorsqu'elle n'a pour se retenir que ce langage où elle s'éteint, se fixe? » (Blanchot, 1973, p. 47)

On le voit, pour Blanchot, la parole qui donne naissance à l'écriture n'est pas de celles qui se laissent cerner. Elle relève, fondamentalement, de l'insaisissable, de ce dont on ne peut rien dire. Peut-être, écrit-il, la source du poème n'est-elle que l'intériorité du poète dont elle manifeste le jaillissement : «Intime est l'image, parce qu'elle fait de notre intimité une puissance extérieure que nous subissons passivement : en 
dehors de nous, dans le recul du monde qu'elle provoque, traîne, égarée et brillante, la profondeur de nos passions. » (1955, p. 356) Peut-être, les mots qui viennent à celui qui écrit ne sont-ils que l'expression de l'influence qu'a eue sur lui un événement dramatique, influence trahie par l'oubli qui agit, dans ce cas, comme une mémoire en négatif : « Ce n'est pas toi qui parleras; laisse le désastre parler en toi, fût-ce par oubli ou par silence. » (1980, p. 12) Si le retrait de l'auteur est, pour Blanchot, la condition de la création, celui-ci s'avère dès lors en définitive condamné à l'échec car l'oubli, pour sa part, ne peut être oublié, et replace inévitablement celui qui écrit au centre de la scène.

Ces éléments, comme ceux qui précèdent, interrogent le produit de la création littéraire. Si la création, pour Blanchot, est pur récit sans objet, et/ou récit d'une expérience dont, par définition, elle ne peut rendre compte, et/ou simple écho subjectif d'un événement oublié, on peut s'interroger sur l'intérêt qu'il a accordé dans son œuvre à la littérature, et au nom qu'il a donné à l'inspiration : pourquoi, si celle-ci est à ce point vaine, l'appelle-t-il une parole, avec la portée performative que ce terme présuppose, et non un simple bavardage? Une chose est sûre : cette parole dont parle Blanchot, celle qui parvient à l'écrivain lorsqu'il se met à l'écoute de l'inaudible, est une parole qui ne renvoie pas à un propos à la manière d'un mot - d'un signifiant - appelé pour désigner une réalité précise - un signifié. Dans la mesure où ce qui la fonde est, nous dit-il, le néant, et/ou de l'ordre de l'indicible, et/ou simple écho subjectif d'un événement oublié, cette parole s'apparente en définitive au silence. Un silence qui, cependant, ne cesse jamais, dans l'œuvre de Blanchot, d'apparaître avec la force et la majesté d'une parole à écouter. 


\section{Derrière les mots, le plein}

Lorsque Blanchot écrit que l'œuvre « n'apporte ni certitude ni clarté » (1955, p. 299), il ne dit pas qu'elle n'a pas de valeur pour autant. L'écrivain, écrit-il, « appartient à un langage que personne ne parle, qui ne s'adresse à personne, qui n'a pas de centre, qui ne révèle rien. [...] Là où il est, seul parle l'être - ce qui signifie que la parole ne parle plus, mais est, mais se voue à la pure passivité de l'être. »(1955, p. 17) Dans cet extrait, Blanchot déplace l'accent habituellement posé sur le fruit de l'acte de parler - les paroles prononcées et leur sens - pour le porter sur l'activité même d'énoncer. Il s'agit, nous dit-il, d'une " affirmation ininterrompue », un " murmure géant », une « profondeur parlante »... Manifestement, cela parle, on ne peut en douter. Mais qu'est-ce que cela raconte? Rien, nous dit Blanchot. Ou plutôt, tout. Rien de précis, mais la possibilité même de dire. Là, écrit-il, « la parole ne parle pas, elle est, en elle rien ne commence, rien ne se dit, mais elle est toujours à nouveau et toujours recommence. » $(1959$, p. 263)

L'être, cet au-delà des apparences dont on ne peut témoigner qu'en perdant la voix, est le lieu du dévoilement : là, « les choses s'offrent dans la fécondité inépuisable de leur sens que notre vision habituellement ignore » (1955, p. 198). L'écriture « n'est pas une simple tromperie, elle est le dangereux pouvoir d'aller vers ce qui est, par l'infinie multiplicité de l'imaginaire » (1959, p. 119). Il y a bien, ici, l'idée d'une promesse. C'est d'une introduction, presque d'une initiation qu'il s'agit ici. Une ouverture à une forme de plénitude. La parole qu'entend l'écrivain semble l'introduire au mystère de sa propre existence : " il semble qu'en elle la profondeur parle, et l'inouï se fasse entendre. À chacun [...] elle 
semble dire ce qui pourrait lui être le plus proche. » (1959, p. 266) Quelque chose se dit, quelque chose qui concerne la possibilité même de dire, et le fait que cela se dise semble apporter la promesse de pouvoir tout dire. Cette promesse, en elle-même, a la valeur de l'inespéré.

Ce n'est plus de vide, de leurre qu'il est question ici, mais $\mathrm{au}$ contraire de quelque chose qui se fait entendre et du sentiment qu'a Blanchot, dans certains détours de son œuvre, que ce quelque chose a un caractère originel : " Il semble [...] que si l'on garde contact avec elle, [...] tout ce qui sera dit appartiendra à la pureté de l'origine. » (1955, p. 242) L'écrivain, à cet égard, apparaît comme un passeur. Il introduit à un autre niveau de perception, à une connaissance plus dense du monde. Certes, nous l'avons vu, ce à quoi l'écriture donne accès n'est pas de l'ordre de contenus qui se présenteraient comme des réponses à des questions posées. C'est cependant bien d'une énonciation qu'il est question ici.

Comme l'a montré Anzieu, de nombreux créateurs partagent le sentiment d'avoir accès, par leur art, à un au-delà de la réalité entretenant avec le(s) sens - l'essence - des choses un rapport privilégié. Loreau, à cet égard, a souligné cependant combien le sens que véhicule l'œuvre d'art cependant est antérieur à la signification. Plutôt que du sens constitué, il relève de la possibilité même de donner sens :

Le sens est un processus de limitation progressive de l'infini des possibles, un mouvement de polarisation des possibles vers une forme; à ce titre, il représente la phase d'éclosion d'une signification. À l'inverse, la signification est un sens venu à l'achèvement, elle est au sens ce que l'immobilité est au mouvement. En tant qu'elle est liée à la fois à l'apparition de la pensée et à ce qui la conteste, la création d'art comme création 
évolue donc dans le sens, elle prend fin avec l'apparition d'une structure comme signification. [...] Ni le produit ni l'apparu ne l'intéresse, seul la concerne le processus de production, d'apparition. (1998, p. 197-198)

Nous retrouvons dans cet extrait l'intérêt porté par Loreau à la création en tant que processus, dont nous avons parlé plus haut. En tant que relation du créateur avec ce qui n'a pas de signification constituée, la création, pour lui, prend place dans le sens, c'est-à-dire " dans le parcours orienté non encore signifiant qu'est le tracement »(1998, p. 186). Le geste créateur, en tant qu'il donne à exister des figures qui ne lui préexistent pas, montre ce qu'il appelle « l'opération génératrice du monde », ou encore " la condition de possibilité de ce qui est en général » (1998, p. 186). Cette approche de la création, dans la mesure où elle aborde celle-ci comme un acte et comme un processus d'ouverture au sens plutôt que comme une offre de signification, a des résonances avec la manière dont Blanchot traite la question - pour autant que l'on puisse comparer ces pensées qui s'appuient sur des pratiques artistiques différentes, les œuvres auxquelles Loreau s'est intéressé (celles de Dubuffet, de Dotremont, d'Alechinsky, etc.) s'inscrivant en effet dans un rapport particulier à la création où le tracé, le geste, célèbrent la spontanéité. Cette approche fait également écho aux travaux d'Alain Didier-Weill, pour qui l'effet d'une œuvre d'art est de l'ordre de la signifiance, c'est-à-dire du « faire sens ». Elle nous met en présence avec un réel qui n'a pas été ligoté par le langage, c'est-à-dire avec un réel signifiant. ${ }^{2}$

2 Il y aurait matière à développer les continuités entre ce qui, pour Lacan, caractérise le réel, et cet espace originel, en-deçà de la signification, dont parle Blanchot lorsqu'il évoque ce avec quoi entre en contact celui qui écrit. 
Parce qu'il est en relation avec la source du dire, l'écrivain, écrit Blanchot, a une responsabilité. Écrire, ce n'est pas se mettre passivement à l'écoute du monde. C'est se donner pour tâche de transmettre la parole de ce dernier. Cette tâche prend, chez lui, la forme d'un véritable sauvetage : "Chaque homme est appelé à recommencer la mission de Noé. Il doit devenir l'arche intime et pure de toutes choses, le refuge où elles s'abritent. » (1955, p. 181). L'écriture doit donner accès à « la terre promise de la vérité » $(1959$, p. 183) En cela, la vision de Blanchot est proche de la manière dont Bazaine définit la mission du peintre :

Le peintre ne vogue pas, ravi, sur un monde bien fini : il participe à sa naissance, mêlé aux sources et aux profondes racines, il suit son cours, la force des choses l'emporte. [...] Le peintre s'efforce, inlassablement, de sauver le monde en le ressuscitant, de laisser pourrir en lui cette graine fabuleuse pour restituer un monde d'au-delà de la mort, un espace inaltérable, une lumière d'au-delà de l'ombre. (1973, p. 89)

Pour Blanchot, le sauvetage dont il est question est, bien sûr, celui d'une parole précieuse, voire sacrée, dont l'écrivain se fait l'héritier; mais il répond, également, à un besoin intime de l'écrivain à qui l'œuvre achevée apporte un sentiment d'apaisement. Elle fait taire « cette parole errante qui ne peut prendre fin, [et qui] est la longue nuit de l'insomnie » (1955, p. 246). Ce tête-à-tête avec la parole du monde est, on le voit, à la fois de l'ordre du sacrifice - sacrifice de celui qui, pour entendre l'inaudible, s'exile de lui-même - et de la délivrance. Cependant, nous l'avons vu, dès que l'écriture cesse la parole se tait elle aussi, privant l'écrivain de cette terre promise loin de laquelle il semble que rien, pour lui, n'a de valeur. Tel Sisyphe il est donc appelé, continuellement, à se remettre à l'ouvrage. 
L'œuvre de Blanchot montre combien complexe est le rapport que l'écriture entretient avec cette parole qui la fonde, dont nous venons de voir qu'elle était à la fois creuse, et pleine; promesse de sens, et ce dont on ne peut rien dire. On ne s'étonne guère que le récit, par conséquent, tombe toujours à côté de ce qu'il vise :

L'écriture : une flèche visant le vide - l'anachronique du futurpassé - et tombant toujours trop tôt, dans le trop plein d'un passé accablant, d'un futur avenir ou bien encore, ce qui est pis, dans la plénitude d'un présent qui transforme tout en écrit riche de ressources et de vie. (1973, p. 169)

Un des mérites de sa démarche est d'avoir mis le doigt sur cette ambiguïté propre aux œuvres littéraires qui, à la fois, livrent le réel et le défigurent, dans le décalage qui toujours sépare l'action de l'acte. L'écriture, avec lui, prend place dans un espace intermédiaire où quelque chose se passe ou, tout au moins, pourrait se passer.

\section{Par-delà les mots qui se cherchent, quelque chose se dit}

L'expression, et notamment l'expression artistique, est tournée vers le déploiement de celui qui crée. Centrée sur lui, elle vise à le révéler. C'est ainsi que nombre d'ateliers d'expression artistique à visée thérapeutique se définissent aujourd'hui : comme des espaces dans lesquels des personnes, en souffrance ou non, sont invitées à utiliser, en toute liberté, des couleurs, de la matière, des sons, des mots, des mouvements, pour laisser surgir les pensées et/ou émotions qui les habitent. S'inspirant de la même idée qu'ils ont empruntée en sens inverse, certains se sont permis d'emprunter les grilles de la psychopathologie pour analyser les œuvres créées par des artistes, et en faire un 
diagnostic. On entend ainsi, dans certains lieux, que tel auteur fut pervers, tel autre psychotique, tel autre encore épileptique... Mais que dit-on, avec ces étiquettes, des œuvres en question? Quelle place accorde-t-on au réel dont chacune d'elles témoigne?

La création, nous l'avons vu, suit un mouvement bien différent de l'expression : il ne s'agit pas pour celui qui crée de projeter sur une matière une part de lui-même, mais au contraire de se mettre en suspens, dans le travail créateur, afin de pouvoir entendre et communiquer quelque chose qu'il éprouve comme venant d'ailleurs. C'est en tout cas ainsi que Blanchot en témoigne et, nous l'avons vu, il n'est pas le seul. Quand bien même cette approche ne saurait être considérée comme la seule valable, quand bien même cet éprouvé ne serait qu'un leurre, il ne mérite pas d'être ignoré pour autant.

Les artistes, on le sait, ne sont pas les seuls à s'être interrogés sur la source de l'imagination. Jung, qui a consacré de nombreuses recherches aux images qui surgissent dans les rêves et dans la création, a développé le concept d'inconscient collectif. Ce dernier terme montre combien, pour lui, de nombreuses images, qu'il appelle archétypales, ont une portée universelle (voir Jung, 1989, et von Franz, 1995). Gilbert Durand (1969), à sa suite, s'est à son tour élevé contre les tentatives de réduire les productions de l'imaginaire à des pulsions subjectives. Il a repris à Jung le concept d' " archétype », dont il a renouvelé le sens. Pour l'un comme pour l'autre, l'imaginaire est pourvu d'un dynamisme propre, et ses productions ne peuvent être appréhendées par la seule entrée de la psychologie individuelle. Quant à Alain Didier-Weill, dont la pensée est grandement inspirée par l'œuvre de Lacan, c'est 
du côté du réel, entendu comme " celui que le refoulement originaire institue comme un incognito radical » (2003, p. 82), qu'il a situé la source de l'art.

Nous voyons combien, dans différentes traditions de pensée, est envisagée l'existence d'une réalité autonome où l'inspiration créatrice trouve sa source, avec laquelle, dans certaines conditions, on peut entrer en contact. Dans cette perspective, l'œuvre d'art fait fonction de témoin. Elle élève à la lumière du jour des bribes de ce qui d'ordinaire échappe à la connaissance, comme autant d'ouvertures sur un univers parallèle dont l'artiste, notamment, aurait la clé. Il s'agit d'un mouvement inverse de celui de l'expression - même si, d'une certaine manière, elle y contribue, à l'image du retour de l'oubli dont parle Blanchot. Dans cette optique, l'expression individuelle, aussi manifeste soit-elle, ne caractérise l'œuvre d'art qu'au titre de reste involontaire, l'écume laissée sur la plage par le mouvement de la mer. ${ }^{3}$ Certes, à l'instar des mots que l'on prononce et qui en disent sur nous bien plus que ce que l'on croit, l'œuvre parle de celui qui crée. Elle reflète son regard sur le monde, celui de son époque, et nombreux sont les critiques d'œuvres littéraires qui ont creusé ce sillon et cherché à mettre au jour les continuités entre le créateur et l'œuvre créée. L'œuvre nous instaure en voyeurs. Voyeurs de l'insu d'un auteur qui, en voulant nous montrer le monde, nous livre en même temps le regard avec lequel il l'a regardé. Cependant, prenons l'image du chien qui, lorsque l'on montre du doigt, suit le doigt et non l'objet montré : est-ce ainsi qu'une œuvre doit

\footnotetext{
${ }^{3}$ La complexité des relations entre création et thérapie est traitée de manière admirable dans l'ouvrage de Jean Florence intitulé Art et thérapie. Liaison dangereuse? (Bruxelles, Facultés Universitaires Saint-Louis, 1997).
} 
être abordée? C'est là, certes, une porte d'entrée. Une porte d'entrée que Blanchot connaissait :

il y a [...] une manière classique de décrire l'expérience littéraire, où l'on voit l'écrivain se délivrer heureusement de la partie sombre de lui-même en une œuvre où elle devient, comme par miracle, le bonheur et la clarté propre de l'œuvre et où l'écrivain trouve un refuge et, mieux encore, l'épanouissement de son moi solitaire en une communication libre avec autrui. C'est là ce qu'a affirmé Freud, en insistant sur les vertus de la sublimation et par cette confiance si émouvante qu'il avait gardée dans les pouvoirs de la conscience et de l'expression. Mais les choses ne sont pas toujours aussi simples [...] (1959, p. 261-262)

Cependant, il ne s'en est pas contenté et l'on comprend, à le lire, pourquoi. S'il s'interroge sur ce qui, dans une œuvre littéraire, parle de son auteur, il ne l'appréhende pas comme un potentiel levier de libération du sujet à l'égard de ce qui le fait souffrir, au contraire. C'est sous les traits d'une tache aveugle que ce souvenir oublié est présenté dans ses écrits; une tache qui s'apparente à un échec, témoin d'une rencontre ratée. Car pour Blanchot, nous l'avons vu, ce que vise celui qui écrit n'est pas de se raconter lui-même. L'écriture est un acte par lequel l'écrivain tente d'approcher la pure présence. L'œuvre qui en découle témoigne de cette rencontre, et c'est là sa raison d'être : laisser entrevoir le sacré comme innommable. Ce qui compte,

c'est peut-être la littérature, mais non pas comme une réalité définie et sûre, un ensemble de formes, ni même un mode d'activité saisissable : plutôt comme ce qui ne se découvre, ne se vérifie ni ne se justifie jamais directement, dont on ne s'approche qu'en s'en détournant, qu'on ne saisit que là où l'on va au-delà, par une recherche qui ne doit nullement se préoccuper de la littérature, de ce qu'elle est « essentiellement ", mais qui se préoccupe au contraire de la réduire, de la neutraliser ou, plus exactement, de descendre, par un 
mouvement qui finalement lui échappe et la néglige, jusqu'à un point où ne semble parler que la neutralité impersonnelle. (1959, p. 243)

En d'autres mots, par-delà ce que l'histoire singulière de son auteur donne à lire, c'est bien davantage le silence de l'œuvre qui mérite l'attention. L'endroit où elle sidère, où elle captive le regard. À travers ce silence, un espace s'ouvre. Quelque chose se dit dont l'auteur ne peut rien dire. Or, cette dimension du rapport du créateur à quelque chose qui ne lui appartient pas, quelque chose qui lui résiste, est précisément la dimension de la création qui est évacuée lorsqu'on assimile celle-ci à de l'expression. On peut se demander, dès lors, si l'engouement actuel pour les ateliers d'expression artistique ne parle pas moins de la création en tant que telle que des aspirations de ceux et celles qui la regardent de loin. Dans une société où l'expression de soi, l'authenticité ont pris la place de véritables idéaux, ce n'est sans doute pas un hasard, en effet, si l'expression artistique a la cote. Ce n'est probablement pas un hasard non plus si la dimension non expressive de la création, celle qui oblige l'artiste à se décentrer, est si peu évoquée.

\section{Vers une approche de la création comme ouverture}

Avec Blanchot, l'écriture est de l'ordre d'une rencontre : une rencontre avec l'insaisissable; une rencontre insaisissable. L'impression qui se dégage de ses écrits est celle d'une tension permanente, dans son expérience de l'écriture, ou tout au moins dans le regard qu'il porte sur elle, entre parole et silence, présence et absence. Cette tension donne l'idée d'un flux, un mouvement, un espace dans lequel des formes naissent constamment et constamment se dissolvent. La pensée de 
Blanchot, en mouvement elle aussi, est à l'image de ce dont elle traite. Sans cesse surgissent des contradictions, des ouvertures qui relancent le sens dans une nouvelle direction. Et cette abondance de possibilités interprétatives est au plus près de ce qu'il tente de décrire : «Au niveau du monde, l'ambiguïté est possibilité d'entente; le sens s'échappe toujours dans un autre sens; le malentendu sert à la compréhension, il exprime la vérité de l'entente qui veut que l'on ne s'entende jamais une fois pour toutes. » $(1955$, p. 358)

L'ambiguïté, cependant, implique un renoncement : le renoncement à la possibilité de faire tenir les choses dans un discours qui les contourne et les clôture. Il est certes plus facile de penser, par exemple, que la création est le simple reflet du vécu d'un auteur. Car alors on peut, pour l'aborder, se tenir à une rampe : celle qu'offre l'histoire du sujet ou, tout au moins, ce que l'on croit en savoir. Cette tendance à réduire la création à une démarche expressive, qui est dans l'air du temps, occulte, nous l'avons vu, une part importante de ce qui est en jeu : elle masque la dimension expérientielle de l'acte créateur en tant qu'épreuve, combat de l'artiste avec un matériau qui lui résiste, à l'image de ce réel avec lequel, par orgueil, il tente, tel Ulysse accroché à son mât, d'entrer en relation. Quelque chose, nous dit-il, parle, et toute la difficulté réside en la manière dont peut être nommée cette parole aujourd'hui. En tant que philosophe, Blanchot pose la question en termes de réel/irréel. Cela le conduit à s'interroger sur le statut ontologique des images qui nourrissent l'inspiration de celui qui écrit. D'autres, certainement, auraient emprunté une autre voie. L'intérêt, cependant, réside peut-être moins dans les termes utilisés que dans l'accent posé par Blanchot sur le vécu qui, à juste titre, mérite d'être ramené au centre de l'attention : celui de la 
création en tant que processus qui toujours en partie échappe à son auteur et qui, parce qu'il lui échappe et en ce qu'il lui échappe, ouvre une brèche dans la réalité. L'œuvre d'art, pardelà la vision qu'elle livre, offre un regard. Elle ouvre à la possibilité de voir. Un des mérites de Blanchot est de l'avoir rappelé.

\section{Bibliographie}

ANTONIOLI, Manola. 1999, L'écriture de Maurice Blanchot. Fiction et théorie, Paris, Kimé.

ANZIEU, Didier. 1981, Le corps de l'œuvre. Essais psychanalytiques sur le travail créateur, Paris, Gallimard, coll. «Connaissance de l'inconscient».

BAUCHAU, Henry. 2000, L'Écriture à l'écoute, Arles, Actes Sud, coll. « Essais littéraires ».

BAZAINE, Jean. 1973, Exercice de la peinture, Paris, Seuil, coll. «Pierres vives ».

BLANCHOT, Maurice. 1955, L'espace littéraire, Paris, Gallimard, coll. «Idées ».

—. 1959, Le livre à venir, Paris, Gallimard.

—. 1973, Le pas au-delà, Paris, Gallimard.

—. 1980, L'écriture du désastre, Paris, Gallimard. 
—. 2002 [1992], Une voix venue d'ailleurs. Sur les poèmes de Louis-René des Forêts, Paris [Plombières-les-Dijon], Gallimard [Ulysse/Fin de siècle].

COLLIN, Françoise. 1971, Maurice Blanchot et la question de l'écriture, Paris, Gallimard, coll. « Le chemin ».

DIDIER-WEILL, Alain. 2003, Lila et la lumière de Vermeer. La psychanalyse à l'école des artistes, Paris, Denoël, coll. «L'espace analytique».

DURAND, Gilbert. 1969, Les structures de l'imaginaire; introduction à l'archétypologie générale, Paris, Bordas, coll. "Études supérieures».

FLORENCE, Jean. 1985, Ouvertures psychanalytiques. Philosophie, arts, droit, psychothérapie, Bruxelles, Facultés universitaires Saint-Louis.

-. 1997, Art et thérapie. Liaison dangereuse?, Bruxelles, Facultés universitaires Saint-Louis.

JUNG, Carl Gustav. 1989, Métamorphoses de l'âme et ses symboles, Genève, Éditions Gerorg.

LANNOY, Jean-Luc. 2005, "Voir, c'est peut-être oublier de parler », dans Arthur COOLS et al. (dir.), Maurice Blanchot, la singularité d'une écriture, Louvain-La-Neuve, Les Lettres romanes, p. 167-189.

LOREAU, Max. 1998, De la création : peinture, poésie, philosophie, Bruxelles, Labor, coll. «Espace Nord».

RABATÉ, Dominique. 2005, « Maurice Blanchot et l'expérience littéraire ", dans Arthur COOLS et al. (dir.), Maurice Blanchot, la singularité d'une écriture, Louvain-La-Neuve, Les Lettres romanes, p. 7-16.

VON FRANZ, Marie-Louise. 1995, L'interprétation des contes de fées, Paris, Albin Michel. 


\title{
Résumé
}

D'où vient à l'écrivain ce qu'il écrit ? Quel est ce avec quoi, par l'écriture, il entre en relation ? Cette question est au cœur de la pensée de Blanchot. Dans la réponse qu'il lui apporte, Blanchot accorde une place centrale à l'expérience d'une rencontre de celui qui écrit avec un au-delà de la réalité visible, qui prend la forme d'une parole. En écho à la manière dont des artistes et des psychanalystes parlent de la création aujourd'hui, son approche nous conduira à interroger la différence entre création et expression.

\begin{abstract}
Where does the source of writing lie? What is this which, through writing, the writer has encountered? This question lies at the core of Blanchot's thought. In his answer, Blanchot gives a central role to the experience of an encounter between the person who writes and something beyond visible reality which takes the form of a speech. His approach, which echoes how some artists and psychoanalysts look at creation today, will enable us to question the difference between creation and expression.
\end{abstract}

